\title{
Experimental evidence of three-dimensional acoustic propagation caused by nonlinear internal waves
}

\author{
Scott D. Frank ${ }^{\text {a) }}$ \\ Department of Mathematical Sciences, Rensselaer Polytechnic Institute, \\ 110 8th Street, Troy, New York 12180 \\ Mohsen Badiey \\ Ocean Acoustics Laboratory, College of Marine Studies, University of Delaware, \\ Newark, Delaware 19716 \\ James F. Lynch \\ Woods Hole Oceanographic Institution, Woods Hole, Massachusetts 02543 \\ William L. Siegmann \\ Department of Mathematical Sciences, Rensselaer Polytechnic Institute, \\ 110 8th Street, Troy, New York 12180
}

(Received 30 September 2004; revised 5 May 2005; accepted 5 May 2005)

The 1995 SWARM experiment collected high quality environmental and acoustic data. One goal was to investigate nonlinear internal wave effects on acoustic signals. This study continues an investigation of broadband airgun data from the two southwest propagation tracks. One notable feature of the experiment is that a packet of nonlinear internal waves crossed these tracks at two different incidence angles. Observed variations for the lower angle track were modeled using two-dimensional parabolic equation calculations in a previous study. The higher incidence angle is close to critical for total internal reflection, suggesting that acoustic horizontal refraction occurs as nonlinear internal waves traverse this track. Three-dimensional adiabatic mode parabolic equation calculations reproduce principal features of observed acoustic intensity variations. The correspondence between data and simulation results provides strong evidence of the actual occurrence of horizontal refraction due to nonlinear internal waves. (C) 2005 Acoustical Society of America. [DOI: 10.1121/1.1942428]

PACS number(s): 43.30.Zk, 43.30.Pc, 43.30.Es [AIT]

Pages: $723-734$

\section{INTRODUCTION}

Acoustic interaction with nonlinear internal waves in shallow-water regions has received significant attention in recent years. Nonlinear internal wave packets are known to affect amplitude ${ }^{1}$ and phase ${ }^{2}$ of single frequency and broadband acoustic signals at $200 \mathrm{~Hz}$ and above. For example, an early paper explains anomalous frequency dependent transmission losses using classical wave-wave interactions, ${ }^{1}$ with resonance occurring when a horizontal wavenumber of a nonlinear internal wave packet is close to a difference between wave numbers of dominant acoustic modes. Recently a theoretical study generalized this condition to relate acoustic wavenumber differences to peaks in the horizontal wavenumber spectrum of an internal wave packet. ${ }^{3}$ Other energyexchange mechanisms are discussed in a recent review. ${ }^{4}$

The SWARM 95 experiment was a multi-institutional effort which acquired high quality environmental and acoustic data. ${ }^{5}$ Two of its goals were to observe nonlinear internal waves and to understand and describe their effects on acoustic signals. For instance, results from this experiment show that arrival time variations ${ }^{6}$ and mode amplitude

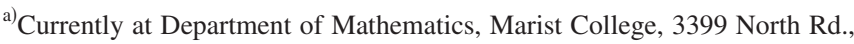
Poughkeepsie, NY 12601; electronic mail: scott.frank@marist.edu
}

decorrelation $^{7}$ of broadband transmissions occur when internal wave packets intersect propagation paths. Numerical studies investigate mode coupling caused by range dependence in nonlinear internal wave packets ${ }^{8,9}$ and the additional influence of a diffuse internal wave field on single frequency acoustic signals. ${ }^{10}$

For six days during the SWARM experiment the R/V Cape Hatteras was southwest of the two vertical linear arrays (VLAs). A 20 in. ${ }^{3}$ Bolt airgun was suspended at various depths from the Cape Hatteras during this time. On 4 August 1995, while the source was at $12 \mathrm{~m}$ depth, a packet of strong nonlinear internal waves crossed the two acoustic propagation tracks with different incidence angles. If the wave fronts are assumed planar, then one angle was near $45^{\circ}$, and the second was near the ray theory estimate of total internal reflection. Acoustic data from both tracks show significant variations while the nonlinear internal wave packet traverses the region, and some features of the variations are correlated with those of the nonlinear internal wave packet. However, acoustic observations from the two tracks do show differences that represent azimuthal dependence of the acoustic field (when viewing from cylindrical coordinates centered on the source). Two-dimensional (2D) parabolic equation (PE) calculations have been used to illustrate ${ }^{11}$ this azimuthal dependence on pulse shape and amplitude. Prior to SWARM, 
Rubenstein and Brill ${ }^{12}$ used a 2D PE to model observed $400 \mathrm{~Hz}$ continuous wave intensity variations at a horizontal array caused by an internal wave packet. These computations accurately represented the presence of acoustic features and their phase speeds, but the authors concluded the model variations did not have sufficient amplitude. Since SWARM, other 2D calculation studies of azimuthal sound-speed profile dependence from internal waves have emphasized multiple resonance effects ${ }^{13}$ and proposed the idea that internal waves cause acoustic focusing. ${ }^{14}$ The international experiment ASIAEX, recently performed in the South and East China Seas, includes investigation of azimuthal-dependent variability. ${ }^{15}$

Even though azimuth-dependent acoustic effects have usually been examined with $2 \mathrm{D}$ (or $N \times 2 \mathrm{D}$ ) propagation techniques, genuinely three-dimensional (3D) mechanisms have been conjectured for some time and have received increasing attention recently. Theoretical studies ${ }^{16,17}$ suggest strong horizontal sound-speed gradients, as introduced by nonlinear internal waves, could cause significant refraction of horizontal acoustic rays. In the Barents Sea, acoustic phase variations were observed on a horizontal array while nonlinear internal waves were believed present. ${ }^{2}$ Computational investigations also concluded that 3D effects of nonlinear internal waves should be observable via intensity $\operatorname{loss}^{3,18}$ or beamforming effects, ${ }^{19}$ although under certain conditions these effects may be minimal. ${ }^{20}$ Only small horizontal refraction was demonstrated theoretically for deepwater situations involving, for example, strong eddies and currents. $^{21,22}$

This paper contains three principal contributions. First, the dominant signals generated by the airgun source are broadband pulses below $100 \mathrm{~Hz}$, with relatively less energy up to $180 \mathrm{~Hz}$. In contrast, recent studies of acoustic propagation influenced by nonlinear internal waves focus on signals at or above $200 \mathrm{~Hz}$. A comparison of data from the two VLAs suggest that nonlinear internal waves cause much larger intensity variability in these frequency bands when the acoustic propagation direction is nearly parallel to internal wave fronts. Second, a full broadband PE propagation model of the Woods Hole Oceanographic Institution (WHOI) track and data is presented. This model includes a treatment of the airgun source, specification of geoacoustic parameters and ocean sound-speed variability, as well as comparison to a corresponding model for the Naval Research Laboratory (NRL) track. ${ }^{23}$ The calculations here use the same nonlinear internal wave parameters selected for the NRL track, and the consistency between computations and data on two independent propagation paths supports these selections. Finally, time-averaged intensity variations of similar magnitude are shown to occur in computational results that account for horizontal refraction, while results from 2D models do not demonstrate variations with sufficient amplitude. The agreement between experimental data and a 3D computational model for this portion of the SWARM site is noteworthy. The correlation between an observed nonlinear internal wave packet and horizontal refraction effects in both model results and data provide, as far as can be determined, the first strong evidence that horizontal refraction due to nonlinear internal waves can cause significant signal variability in the ocean.

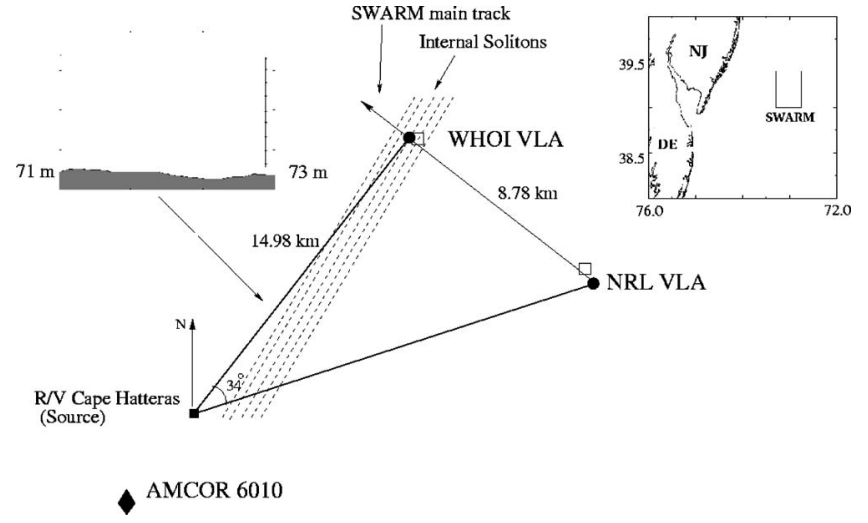

FIG. 1. Geometry of southwest portion of SWARM experiment. Signals from airgun at R/V Cape Hatteras (black square) received by WHOI and NRL VLAs (black circles). Thermistor strings (white squares) associated with each array. AMCOR 6010 site (black diamond) located several kilometers southwest of source. Bathymetry of WHOI waveguide is nearly flat. Two acoustic tracks (dark lines) intersected by internal soliton packet (dashed lines) observed on 4 August 1995.

The Barents Sea study ${ }^{2}$ described observed horizontal acoustic phase fluctuations but did not have internal wave packet observations to correlate with these fluctuations.

The paper is organized as follows. Section II briefly reviews environmental and acoustic data available from the two southwest tracks of SWARM. Significant time-frequency and pulse-averaged intensity variability occur at both arrays. Section III provides internal wave and geoacoustic parameters used for 2D modeling of the track waveguides. Data and simulation comparisons show 2D methods appear unable to model acoustic variability at the WHOI VLA. Some aspects of 3D propagation and the adiabatic mode PE method used for 3D computations are reviewed. Section IV shows that the relatively large variations observed in data can be reproduced by incorporating the mechanism of horizontal refraction. Section V contains a summary and discussion of main results.

\section{EXPERIMENT BACKGROUND}

The full SWARM experiment ${ }^{5}$ was conducted in 1995 off the New Jersey coast (see Fig. 1 inset). Figure 1 shows the southwest tracks which are the focus of this study. Suspended at $12 \mathrm{~m}$ depth from the R/V Cape Hatteras (indicated by a black square in the lower left) was a 20 in. ${ }^{3}$ Bolt airgun which was fired every minute for several hours on 4 August 1995. The source signal was extremely repeatable, with usable bandwidth between 10 and $180 \mathrm{~Hz}$ and large energy peaks at $32 \mathrm{~Hz}$ and several harmonics. ${ }^{11}$

The airgun signal was received by two VLAs, indicated by black circles in Fig. 1. The primary subject of this study is data from the WHOI telemetered array consisting of 16 hydrophones spaced approximately $3.5 \mathrm{~m}$ apart with the top and bottom phones at 14.9 and $67.5 \mathrm{~m}$ depths. This array was suspended in $70.5 \mathrm{~m}$ of water and was about $15 \mathrm{~km}$ from the source. Details of the NRL VLA are provided for comparison. This array consisted of 32 elements spaced $2 \mathrm{~m}$ apart with the top and bottom phones at 21 and $85 \mathrm{~m}$ depths. 
It was suspended in $88 \mathrm{~m}$ of water about $18 \mathrm{~km}$ northeast of the source and $9 \mathrm{~km}$ southeast of the WHOI array, as shown in Fig. 1.

Several packets of nonlinear internal waves passed through the SWARM region on 4 August and were monitored at several locations. ${ }^{6}$ CTD readings performed by the R/V Cape Hatteras provide data about sound-speed profile variations at the source. Two thermistor strings (with five thermistors each) collected temperature data at locations of white squares in Fig. 1. The first was attached to the WHOI VLA and the second was attached to the NRL VLA. In addition the R/V Oceanus was near the WHOI array and used radar images of nonlinear internal wave packets to estimate their bearing. ${ }^{5}$ During a particular $2 \mathrm{~h}$ period on 4 August a large packet of nonlinear internal waves passed between the airgun source and the two VLAs. This packet was recorded by the WHOI thermistor string and observed on CTD readings performed at the R/V Cape Hatteras. It was also observed near the WHOI VLA on radar by the R/V Oceanus. The propagation direction of the packet was estimated from these radar images. Dashed lines in Fig. 1 represent this packet of waves with linear wave fronts. This linear-front idealization is a modeling assumption since satellite images suggest the SWARM region contained a great deal of internal wave activity and that the solitary wave fronts have curvature. ${ }^{5,24}$

For this study we define the incidence angle $\phi$ as the angle from the propagation direction of the acoustic signal to that of the nonlinear internal wave measured positive counterclockwise. Thus, if $\phi=90^{\circ}$ the wave fronts are parallel to the acoustic track and are moving from right to left across the track. Note the different incidence angles for the two acoustic propagation tracks on Fig. 1 , about $45^{\circ}$ for the NRL track and about $85^{\circ}$ for the WHOI track (see Fig. 1 of Ref. 11).

Gabor wavelet transforms of broadband signals influenced by nonlinear internal waves reveal complex variations at the NRL VLA. ${ }^{23}$ Corresponding wavelet transform results are shown in Fig. 2 for phone 2 (19 m depth) of the WHOI VLA. Phone 2 was chosen because its position at about onethird of the water depth records observations of higher mode arrivals. Results from two signals are shown in three-panel figures. The top panel is a normalized representation of the time domain signal, the right panel is the normalized Fourier spectrum, and the large central panel is the graph of a wavelet transform (scalogram). Figure 2(a) shows the 1945 GMT shot, the spectrum for which shows peak frequencies at 32, 64, and $95 \mathrm{~Hz}$. Group velocity curves are visible in the scalogram and most acoustic energy is in the first two modes of the $32 \mathrm{~Hz}$ band and in the late-arriving third mode of the $64 \mathrm{~Hz}$ band. Figure 2(b) shows the 1952 GMT shot, $7 \mathrm{~min}$ later. Its spectrum indicates more high frequency harmonics, confirmed by the scalogram showing at least three modes near $96 \mathrm{~Hz}$ and four or more near $120 \mathrm{~Hz}$. The scalogram also shows most energy in the $64 \mathrm{~Hz}$ band arrives as part of the second mode. These variations in the patterns of energy distribution are quasiperiodic and correlate with the passage of the nonlinear internal wave packet. Similar variations occurred in signals at the NRL array. (a)
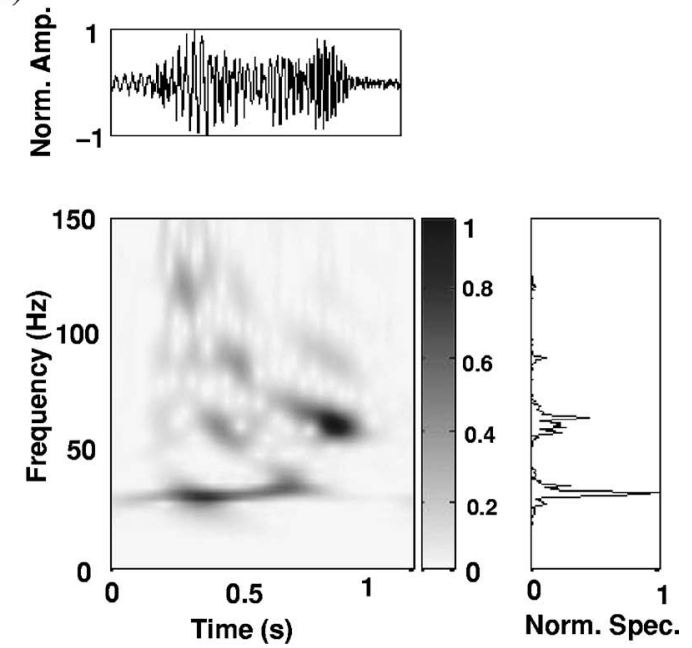

(b)
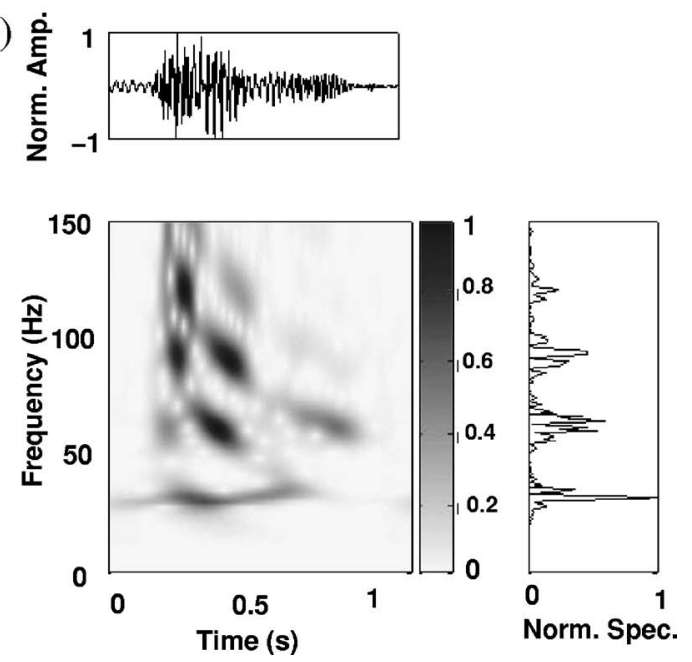

FIG. 2. Time-frequency analysis of WHOI data at phone 2 (19 $\mathrm{m}$ depth). (a) 1944 GMT shot, received time domain signal (top panel). Fourier transform (side panel) shows dominant energy peaks at 32, 64, and $95 \mathrm{~Hz}$. Scalogram (shaded panel) represents Gabor wavelet analysis of signal in top panel. Group velocity curves are visible; two, three, and four modes appear near 32, 64, and $95 \mathrm{~Hz}$. Signal is dominated by $32 \mathrm{~Hz}$ first mode and $64 \mathrm{~Hz}$ third mode energy. (b) 1951 GMT shot (7 min later). Spectrum shows more energy in high frequency bands compared with (a), which is confirmed by scalogram. The $64 \mathrm{~Hz}$ band is now dominated by second mode energy.

The pulse-averaged intensity in $\mathrm{W} / \mathrm{m}^{2}$ of a broadband signal $p(t)$, converted to decibel measure, is

$$
I_{T}=10 \log _{10}\left(\frac{1}{T} \int_{0}^{T} \frac{|p(t)|^{2}}{\rho c} d t\right) \quad \mathrm{dB} \text { re: } 1 \mu \mathrm{Pa},
$$

where $T$ is the the interval of integration, $t$ is time, $\rho$ is water density, and $c$ is an average sound speed. The interval of integration was chosen separately for each array to contain a complete pulse, $1.6 \mathrm{~s}$ for the NRL VLA and $1.2 \mathrm{~s}$ for the WHOI VLA. The quantity $I_{T}$ is calculated for the entire hour of airgun shots beginning at 1901 GMT on 4 August for both arrays. Figure 3(a) shows calculations for the WHOI VLA and Fig. 3(b) for the NRL VLA. All hydrophones on each array are shown with depth-averaged variations indicated by thick curves at the bottom. The "geotime" label is used to emphasize that environmental 


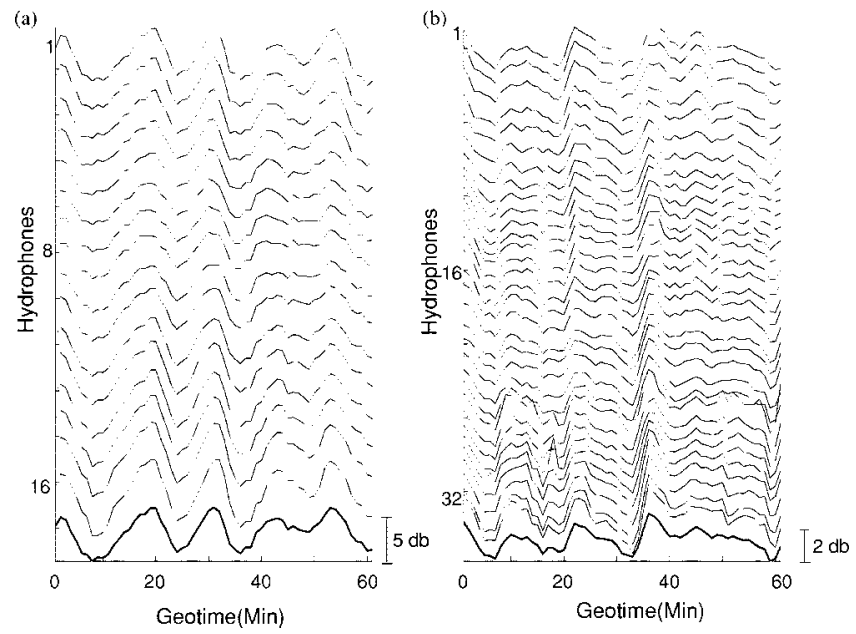

FIG. 3. Intensity $I_{T}$ for each hydrophone in (a) WHOI and (b) NRL VLA vs geotime starting at 1901 GMT. Quasiperiod of variations at each phone is consistent with observed internal waves. Depth-averaged $I_{T}$ (dark curves) shown at bottom. Note amplitude of depth-averaged variations in (a) is nearly twice that in (b).

variations occur on scales much larger than the acoustic pulse. Multiple-decibel variations occur at all hydrophones at each array. The occurrence and phase of these variations is independent of depth, but a weak depth dependence can be seen in the amplitude. The variations are quasiperiodic over about 12-14 min, which are well correlated with observations of the traveling nonlinear internal wave packet. ${ }^{11}$ The maximum amplitude of depthaveraged variations is approximately $5.9 \mathrm{~dB}$ at the WHOI array, which is nearly twice the $3.1 \mathrm{~dB}$ amplitude of oscillations at the NRL VLA. This indicates that the nonlinear internal wave packet has an even greater influence on the acoustics propagating along the WHOI track. Figure 3 has been reproduced from Ref. 23 to allow easy comparison of the difference between the $I_{T}$ variations at each VLA.

\section{MODELING}

The following describes sound-speed profile, geoacoustic, and nonlinear internal wave models used for the analysis of SWARM data. Then results of 2D PE modeling of the broadband signals from the WHOI track are compared with data. Theoretical estimates for several characteristics of $3 \mathrm{D}$ acoustic propagation are obtained. Finally, the method used for 3D simulations is summarized.

\section{A. Environment}

Data from CTD casts taken at the Cape Hatteras while the airgun was firing provided 25 sound-speed profiles at the source location on 4 August $1995 .{ }^{11}$ The average of these profiles is used as a range-independent mean sound-speed profile in the water column. The profile is downward refracting with a $1534 \mathrm{~m} / \mathrm{s}$ upper layer and strong thermocline transition, between 10 and $30 \mathrm{~m}$ depth, to a $1484 \mathrm{~m} / \mathrm{s}$ lower layer. Temperature and salinity data collected by the $\mathrm{R} / \mathrm{V}$ Oceanus during this time period suggests the presence of an upwardly refracting bottom layer along the main track of
SWARM, especially off the shelf break (see Fig. 4 in Ref. 5). Due to the location of the WHOI array and the absence of this feature in the Cape Hatteras data, this study focuses on the effects of the nonlinear internal waves by using the range-independent mean sound-speed profile. Bathymetry data for the WHOI track was obtained from the National Geophysical Data Center. ${ }^{25}$ The nearly flat bathymetry is shown in an inset of Fig. 1.

The nonlinear internal wave packet is assumed to perturb the mean sound speed. As a model of the packet's effect we use a piecewise linear approximation of the first internal wave gravity mode $\Phi_{1}(z)$ multiplied by a sum of six $\operatorname{sech}^{2}$ waves:

$$
\eta(r, z)=\Phi_{1}(z) \sum_{n=0}^{5} A_{n} \operatorname{sech}^{2}\left[\frac{2 \pi\left(r-r_{n}+v_{n} t\right)}{\Lambda_{n}}\right]
$$

where $(r, z)$ are cylindrical coordinates, $A_{n}$ represents the amplitude of the $n$th wave, $\Lambda_{n}$ is its width, $r_{n}$ is its starting position, and $v_{n}$ is its speed. Time $t$ corresponds to geotime in Fig. 3. While packet dispersion can be included in this model by assigning each wave in the packet a distinct $v_{n}$, time-evolution of the packet will not be addressed in this paper. Good estimates of the other parameters can be obtained by matching packet spectral characteristics to those of data. The spectra can be matched well ${ }^{23}$ by using evenly spaced waves with $\Lambda_{n}=140 \mathrm{~m}$ and $r_{n}=2.3 n \Lambda_{n} \mathrm{~m}$, although these certainly are not a unique set of parameters. With the assumption of linear internal wave fronts and track geometry described in Sec. II, these values are projected onto each acoustic track to obtain effective wavelength values of $\Lambda_{\mathrm{NRL}}=195 \mathrm{~m}$ and $\Lambda_{\mathrm{WHOI}}=1600 \mathrm{~m}$ for 2D simulations. In addition, an internal wave packet speed of $v_{n}=v=0.42 \mathrm{~m} / \mathrm{s}$ is used to be consistent with the NRL model. Note that this value is lower than propagation speeds reported on different days of the experiment. However, this value-when projected onto the NRL track-is consistent with observations of this packet at the Cape Hatteras, ${ }^{11,23}$ and was used to model acoustic data from the NRL VLA. It is important to note that in this analysis procedure of the WHOI VLA data, the same water sound speed profiles and nonlinear internal wave parameters are used as for the NRL VLA analysis. Thus, a positive comparison between data and computations on this track will also lend support to the choice of parameters describing this particular nonlinear internal wave packet.

Data from the AMCOR 6010 core is indicated by circles on the dashed-dotted curves in Figs. 4(a)-4(c) for sound speed, attenuation, and density. Distinctive characteristics of this profile are the shallow reflector within $10 \mathrm{~m}$ of the ocean-sediment interface and a low sound speed channel above a deep, high sound speed reflector. The presence of the deep reflector is widely recorded for this region. ${ }^{26}$ To obtain geoacoustic parameters that modeled results at the NRL VLA, the core data were perturbed until acceptable visual matches were achieved between mode amplitudes and relative arrival times of observed data and broadband PE calculations. The light solid curves in Fig. 4 show the geoacoustic profiles that were obtained for the NRL track using a match- 

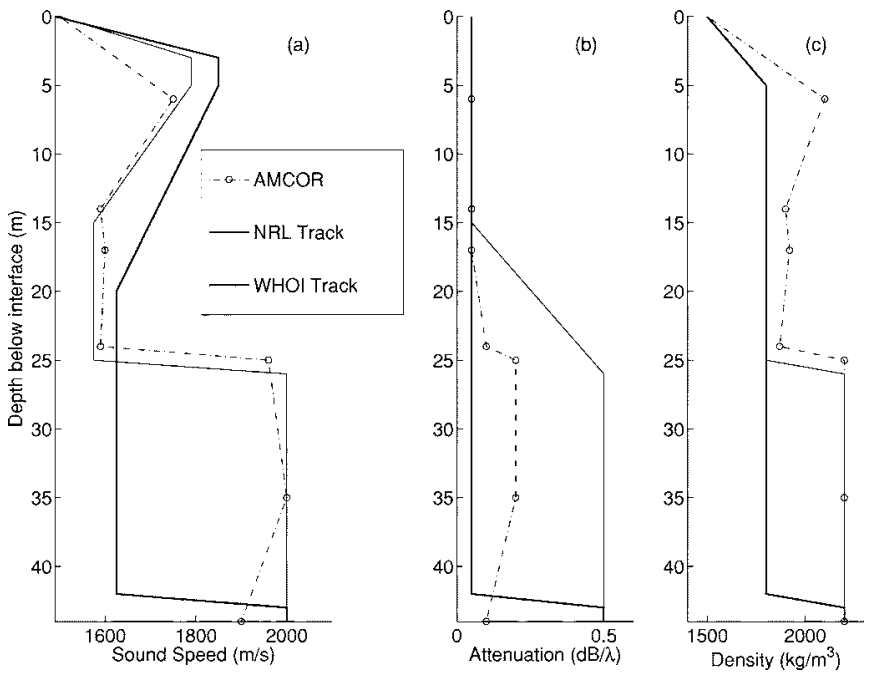

FIG. 4. AMCOR 6010 data (dashed curve with circles), model geoacoustic profiles used in Ref. 23 (solid curve), and model geoacoustic profiles used here (heavy solid curve): (a) sound speed $(\mathrm{m} / \mathrm{s})$, (b) attenuation $(\mathrm{dB} / \lambda)$, and (c) density $\left(\mathrm{kg} / \mathrm{m}^{3}\right)$. Shallow reflector, low sound speed waveguide, and deep strong reflector are preserved in both models. To match modal characteristics in WHOI data, strong reflector must be deeper than indicated by AMCOR.

ing procedure. ${ }^{23}$ However, these parameter values do not accurately reproduce characteristics of the broadband data at the WHOI VLA. In each panel of Fig. 5, the top curve represents broadband data from phone 2 of the WHOI VLA and the middle curve represents PE simulations using geoacous-
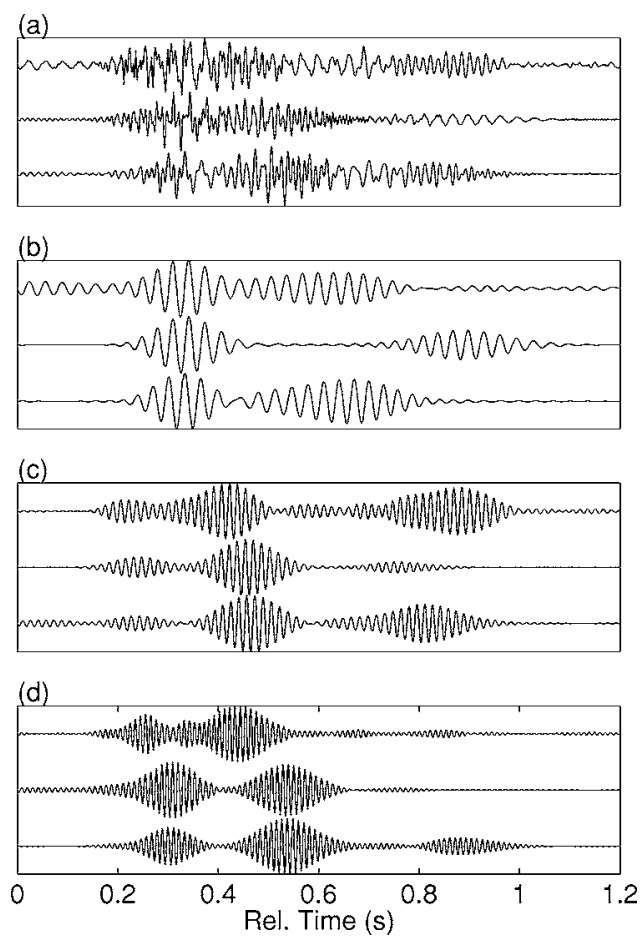

FIG. 5. Comparisons showing modal characteristics among time series of data (top curve in each panel), RAMGEO simulations using Ref. 23 geoacoustic model (middle curves), and simulations using Fig. 4 model (bottom curves). (a) Full frequency band. (b) Results from applying $10 \mathrm{~Hz}$ bandwidth Butterworth filter centered at $32 \mathrm{~Hz}$ to corresponding full band curves in (a). (c) Same, but filter centered at $64 \mathrm{~Hz}$. (d) Same, but filter centered at $95 \mathrm{~Hz}$. In all panels the bottom curves provide better matches of data modal occurrence, dispersion, and strength than the middle curves. tic parameters obtained for the NRL track. Figure 5(a) shows full band signals, while (b), (c), and (d) show signals that have been filtered with a $10 \mathrm{~Hz}$ bandwidth order 10 Butterworth filter centered at 32, 64, and $95 \mathrm{~Hz}$, respectively. The strongest observable differences between the data and the middle curves are the late arrival time of the second mode in the $32 \mathrm{~Hz}$ band [Fig. 5(b)] and the small amplitude of the third mode in the $64 \mathrm{~Hz}$ band [Fig. 5(c)].

Figure 5 implies that the geoacoustic profiles along all or part of the WHOI track differ from those along the NRL track. Of course no claim can be made that the NRL profile parameters describe the actual geoacoustics along that track; these parameters provide only a range-independent representation that is useful for acoustic modeling. It is not surprising that actual geophysical variability along the WHOI track requires a different range-independent representation. Thus, the matching procedure is repeated by making physically based perturbations to the light solid profiles in Fig. 4.

Using geoacoustic profiles shown by heavy solid curves in Fig. 4, the bottom curves in each panel of Fig. 5 are obtained. These curves are more consistent with the data. In particular the arrival time of the second mode at $32 \mathrm{~Hz}$ is much closer to the observations, as is the amplitude of the higher modes at $64 \mathrm{~Hz}$. More detailed agreement could be sought, but this improvement is sufficient for our modeling. The new geoacoustic profiles preserve principal characteristics of the AMCOR profile, but with a deeper strong reflector and a consequently broader soft-sediment channel between the two reflectors. Note that increased attenuation values in the deep reflector are expected since elastic effects are not incorporated explicitly. ${ }^{27,28}$ These bottom parameters will be used for WHOI track models in the remainder of the study. We stress that the profiles in Fig. 4 are not intended as mathematical inversions of the bottom in this region, as that work is being pursued by others. ${ }^{29}$

\section{B. 2D acoustics}

For broadband computations and data analysis, a reasonably faithful representation of the source is essential. As described in Ref. 23, the signal available from the sourcemonitor hydrophone could not be used because it was corrupted by surface and bottom echo returns. A model source representation was developed by adding Gaussian pulses centered at $32 \mathrm{~Hz}$ and several harmonics in the frequency domain so that the spectrum of broadband computations was consistent with data spectra at both VLAs. By using the inverse Fourier transform, a signature was obtained that maintains the impulsive character of the airgun and contains acoustic energy in the appropriate frequency bands. Due to its success modeling data at the NRL array, and to maintain consistency between the two tracks, the identical source signature is used for propagation computations on the WHOI track.

Two-dimensional PE calculations successfully reproduced fluctuations in time-frequency behavior and pulseaveraged intensity at the NRL VLA. ${ }^{23}$ Geoacoustic layers were assumed to be of constant thickness and follow the bathymetry, so the RAMGEO propagation model is 
TABLE I. Column 1 shows Fourier spectrum peak frequencies for nonlinear internal wave packet of 4 August 1995. Internal wave speed of $0.42 \mathrm{~m} / \mathrm{s}$ gives wavenumber values in column 2 and wavenumber values projected onto the WHOI track in column 4.

\begin{tabular}{cccccc}
\hline \hline \multicolumn{3}{c}{ WHOI thermistor data } & & \multicolumn{2}{c}{ WHOI track } \\
\cline { 1 - 3 } \cline { 5 - 6 }$f_{\mathrm{iw}}(\mathrm{mHz})$ & $\kappa_{i w}(\mathrm{rad} / \mathrm{m})$ & $\lambda_{i w}(\mathrm{~m})$ & & $\kappa_{\mathrm{WHOI}}(\mathrm{rad} / \mathrm{m})$ & $\lambda_{\mathrm{WHOI}}(\mathrm{m})$ \\
\hline \multirow{2}{*}{1.32} & 0.0192 & 319 & & 0.00172 & 3660 \\
2.43 & 0.0382 & 173 & & 0.00317 & 1985 \\
3.95 & 0.0620 & 106 & & 0.00516 & 1216 \\
\hline \hline
\end{tabular}

appropriate. ${ }^{30}$ Additionally, it was shown that acoustic wavenumber estimates for the NRL track satisfied the internal wave-acoustic resonance condition

$$
\kappa_{i w} \approx k_{n}-k_{m},
$$

where $\kappa_{i w}$ is an effective peak in the horizontal wavenumber spectrum of internal wave disturbances and $k_{n}$ and $k_{m}$ are wavenumbers of acoustic modes $n$ and $m$. Theory and numerical simulations suggest significant mode coupling occurs when this condition is satisfied, leading to observable variations in strength and modal composition of the signal. ${ }^{1,31}$ Table I shows frequency (column 1) and wavenumber estimates (column 2) of peak locations in the spectrum of the nonlinear internal wave packet of interest. Spectral information was obtained from WHOI thermistor string data. Wavenumbers in column 2 and wavelengths in column 3 are obtained using an internal wave packet speed estimate of $v=0.42 \mathrm{~m} / \mathrm{s}$. Column 4 of Table I shows the effective spectral peak locations when the packet is projected onto the WHOI track using the angle estimate $\phi=85^{\circ}$. Table II shows acoustic wavenumbers for the three lowest dominant frequencies in the airgun signal. These values were calculated using geoacoustic parameters given by the dark curves in Fig. 4 and the normal mode program COUPLE. ${ }^{32}$ Table III shows differences between selected wavenumbers in Table II. From the corresponding table for the NRL track, numerous opportunities for resonant interaction exist from Eq. (3). However, when $\kappa_{i w}$ in Eq. (3) is a value of $\kappa_{\text {WHOI }}$ from Table I, Table III indicates how rare the possibilities for resonance interaction are. Only one wavenumber difference (underlined) is within $10 \%$ of an effective wavenumber spectrum peak. Consequently, we expect that $2 \mathrm{D}$ simulations will not reproduce observed $I_{T}$ variation. Figure 6 shows this situation, with dashed curves representing depth-averaged $I_{T}$ from the data and solid curves representing depth-averaged $I_{T}$ for $60 \mathrm{~min}$ of $2 \mathrm{D} \quad \mathrm{PE}$ simulations using the sound-speed, geoacoustic, and internal wave parameters discussed for an $85^{\circ}$ incidence angle
TABLE II. Acoustic wavenumbers calculated at source using COUPLE for model environment with no internal waves. Three, six, and eight propagating modes occur near 32, 64, and $95 \mathrm{~Hz}$.

\begin{tabular}{llll}
\hline & $32 \mathrm{~Hz}$ & $64 \mathrm{~Hz}$ & $95 \mathrm{~Hz}$ \\
\hline 1 & 0.131 & 0.268 & 0.399 \\
2 & 0.117 & 0.258 & 0.392 \\
3 & 0.108 & 0.244 & 0.381 \\
4 & & 0.240 & 0.366 \\
5 & & 0.220 & 0.364 \\
6 & & 0.204 & 0.345 \\
7 & & & 0.332 \\
8 & & & 0.316 \\
\hline \hline
\end{tabular}

as in Sec. III A. Figure 6(a) shows the calculated $I_{T}$ for computed full band signals, while Fig. 6(b) displays $I_{T}$ of bandpass filtered signals centered at $32 \mathrm{~Hz}$. The data (dashed line) show large oscillations for each frequency band, while the simulations undergo little or no variation. Similar results occur for the 64 and $95 \mathrm{~Hz}$ frequency bands. These results are shown for consistency with the SWARM geometry, and similar graphs are obtained in the case of perpendicular propagation $\left(90^{\circ}\right.$ incidence).

Several possibilities could explain the inability of 2D PE simulations to model the observed variations. Among these are frequency-dependent bottom attenuation in upper sediment layers ${ }^{33}$ or shear processes in the sediment. ${ }^{34}$ These and other possibilities were investigated numerically, but with no success in reproducing variations in the data. In addition a large number of different values were examined for internal wave and geoacoustic profile parameters of the models in Sec. III A, also without obtaining significant acoustic variability. Another possibility is that acoustic scattering out of the vertical propagation plane is not negligible. This implies sound speed variations from the nonlinear internal waves cause significant acoustic horizontal refraction, ${ }^{8}$ which can be investigated using $3 \mathrm{D}$ computational methods.

\section{3D acoustics}

In this section we indicate why $3 \mathrm{D}$ propagation modeling is expected to resolve the mismatch between data and 2D simulations that was discussed in Sec. III B.

We show first that horizontal sound speed gradients in the internal wave environment are sufficient to produce regions of intensity focusing and defocusing over ranges and angular spreads of interest in the SWARM experiment. For simplicity ray theory is used here, since an approach based on adiabatic modes and horizontal rays ${ }^{16}$ is a reasonable approximation to the propagation physics. Also, instead of us-

TABLE III. Differences between selected acoustic wavenumbers from Table II. Comparisons with $\kappa_{\text {WHOI }}$ wavenumbers in Table I shows essentially no opportunities (except for one possibility, underlined) for acoustic mode coupling within the internal wave packet.

\begin{tabular}{lccccccc}
\hline \hline & $k_{1}-k_{2}$ & $k_{1}-k_{3}$ & $k_{2}-k_{3}$ & $k_{2}-k_{4}$ & $k_{3}-k_{4}$ & $k_{3}-k_{5}$ & $k_{4}-k_{5}$ \\
\hline $32 \mathrm{~Hz}$ & 0.0137 & 0.0224 & 0.00876 & & & & \\
$64 \mathrm{~Hz}$ & 0.00936 & 0.0239 & 0.0145 & 0.0187 & 0.00422 & 0.0238 & 0.0196 \\
$95 \mathrm{~Hz}$ & 0.00763 & 0.0184 & 0.0108 & 0.0259 & 0.0151 & 0.0167 & $\underline{0.00161}$ \\
\hline \hline
\end{tabular}




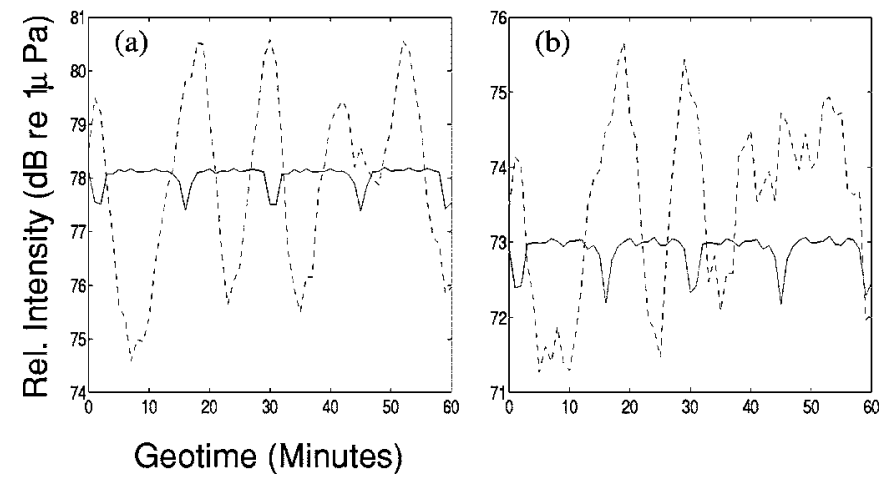

FIG. 6. Depth-averaged intensity variations for data (dashed curves) and 2D PE simulations (solid curves) of WHOI waveguide: (a) full band, and (b) Butterworth filtered band centered at $32 \mathrm{~Hz}$. Relative intensity represents $I_{T}$ converted to $\mathrm{dB}$ re: $1 \mu \mathrm{Pa}$. Simulations are unable to reproduce large variations in WHOI data in any frequency band.

ing a horizontal sound speed channel formed from one of the nonlinear internal waves in Eq. (2), we use a symmetric approximation consisting of piecewise linear segments. The advantage is that ray paths over constant gradient segments are circular arcs. The sound speed profile for a "focusing" case is shown in Fig. 7(a), where $\delta c$ is the maximum change produced by the internal wave over a horizontal distance $W$. The source $S$ is located at horizontal coordinate $y=0$, which we take as the position of the sound speed minimum $c_{\min }$. Consequently the right half of the sound speed profile in the simplified model is given by

$$
c(y)=c_{\min }+\delta c \frac{y}{W}, \quad 0 \leqslant y \leqslant W .
$$

The ray paths shown represent the trapped rays with maximum horizontal excursions, and they determine the angular spread produced by the channel. It follows from Eq. (4) that the radius of these rays is ${ }^{35} R \approx\left(c_{\min } / \delta c\right) W$. From geometry half the distance to the focus point is $L=\sqrt{2 W R}$ since $W / R$ is small. Therefore, the focus distance is $2 W \sqrt{2 c_{\min } / \delta c}$ $\approx 22 \mathrm{~W}$, or about every $3.5 \mathrm{~km}$ for our parameter choices. The total angular spread of the channel is $2 \phi_{\text {crit }} \approx 2 W / L$ $\approx 2 \sqrt{\delta c / 2 c_{\text {min }}} \approx 12^{\circ}$. These estimates provide a preview for simulation results in Sec. IV.

Though the intensity of focusing created will depend on the exact "focusing profile" encountered, we can still estimate the average amount of intensity increase versus range created by soliton ducting by using a simple physical argument. ${ }^{36}$ Specifically, acoustic rays encountering the solitons at angles less than or equal to the critical grazing angle will be trapped between the solitons, which are separated by distance $2 W$ (as in Fig. 7). For these rays, no cylindrical spreading loss will occur. On the other hand, in the absence of soliton ducting, rays at the critical angle or below will suffer cylindrical spreading, subtending an area $\Delta=R \phi_{\text {crit }}$ at range $R$ (note that we implicitly assume the depth dimension $H$ for areas.) By taking the ratio of the areas, we obtain a mean intensity increase due to ducting at range $\mathrm{R}, \Delta / 2 W$ $=R \phi_{\text {crit }} / 2 W$, or about 10 for our parameters. This simple "sonar equation" type calculation gives reasonable agreement to experimentally observed numbers. ${ }^{36}$ (a)

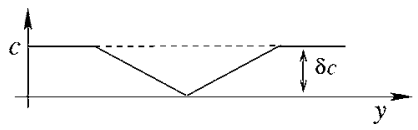

(b)

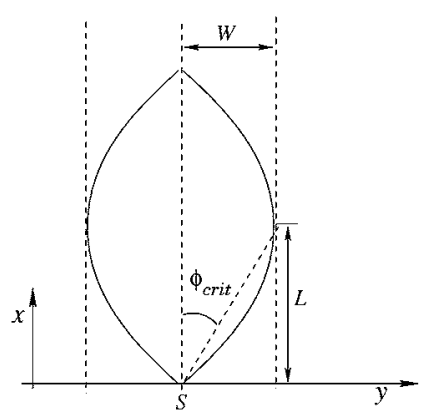

(c)

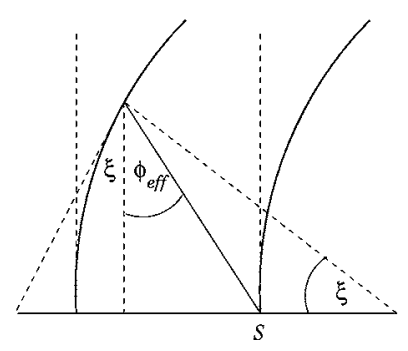

FIG. 7. (a) Idealized representation of horizontal sound-speed variation in focusing waveguide (between two nonlinear internal waves). (b) Variables used for estimate of focal range. Acoustic source is located at $S, W$ is the horizontal distance from middle of trough to peak of nonlinear internal wave, $L$ is half the distance to the focal point, and $\phi$ represents the acoustic angular spread. (c) Schematic for curved nonlinear internal waves. Acoustic source is located at $S$, and $\xi$ is determined by radius of curvature.

The above ducting scenario is a reasonable first approximation to many continental shelf internal wave systems, but does not represent all the possible wave systems seen in nature, and also has an "infinite horizontal correlation length" assumption built in that is obviously an approximation.

One geometry that is commonly seen in nature, when solitons are generated by a submarine canyon or valley, is a circular wave front. For this case, acoustic energy ducted between solitary waves will reflect back and forth from the waves at angles which are alternately increased and decreased by an angle $\xi$. This angle is obtained from the law of sines using $\phi_{\text {crit }}, W$, and the radius of curvature of the internal waves $r_{c}$. The curvature will cause acoustic rays that are pushed above the usual critical grazing angle to leak out of the waveguide, i.e., become untrapped. This results in an "effective critical angle" which is $\phi_{\text {eff }}=\phi_{\text {crit }}-\xi$ for ducted rays in a curved wavefront, as shown in Fig. 7(c).

The finite horizontal correlation length of the internal waves leads to acoustic energy leaking out of the duct between two internal waves to "neighboring ducts," a type of horizontal diffusion process. Though the way this diffusion acts is likely somewhat complicated to model exactly, we can estimate it in a simple fashion. If we assume the model that one duct splits into two ducts of the same area every horizontal correlation length of the waves, then the area subtended by the duct versus range will simply be $\Delta_{\text {diffuse }}$ $=2 W N$, where $N$ is the number of correlation lengths in range and again we have implicitly included the water depth $H$. A slight variant of this can be considered where the en- 
ergy starts its ducting between the leading edge soliton of a wave train and the second wave. In this case, the energy can diffuse only to the back of the wave train, i.e., in one direction, and so the above-quoted area should be reduced by one half. This horizontal diffusion estimate is admittedly crude, but should be able to provide at least order of magnitude numbers for the effect.

\section{Adiabatic mode parabolic equation}

Since 2D full wave calculations cannot reproduce the large $I_{T}$ variations observed at the WHOI VLA, it is necessary to determine if acoustic horizontal refraction can. This section summarizes the adiabatic mode parabolic equation (AMPE), which is an efficient method for solving range- and azimuth-dependent propagation problems. The method relies on the adiabatic mode approximation, so that unlike the PE calculations no coupling of acoustic modes is handled. Examples for 3D bathymetry environments are given in Refs. 37 and 38, and more recently, Ref. 39 suggests using a similar method for internal wave environments. The method computes mode coefficients in a full circular region about the source rather than enforcing boundary conditions on a wedge shaped region. ${ }^{18,20,40}$

The method uses a local mode representation of the Helmholtz equation solution in cylindrical coordinates $(r, \theta, z)$,

$$
p(r, z, \theta)=\sum_{n} \frac{u_{n}(r, \theta)}{\sqrt{k_{n}(r, \theta)}} \psi_{n}(z ; r, \theta),
$$

where the modes $\psi_{n}(z ; r, \theta)$ and wave numbers $k_{n}(r, \theta)$ satisfy the depth operator equation

$$
\left(\frac{\rho}{\alpha} \frac{\partial}{\partial z} \frac{1}{\rho} \frac{\partial}{\partial z} \alpha+k^{2}(r, \theta)\right) \psi_{n}=k_{n}^{2} \psi_{n} .
$$

The factor $\alpha=\sqrt{\rho c}$ is used to conserve energy at vertical interfaces. By substituting Eq. (5) into the Helmholtz equation, using Eq. (6), and neglecting mode coupling terms and wavenumber derivatives, a parabolic equation for the mode coefficients $u_{n}$ can be obtained:

$$
\frac{\partial u_{n}}{\partial r}=i k_{0} \sqrt{1+\frac{1}{k_{0}^{2} r^{2}} \frac{\partial^{2}}{\partial \theta^{2}}+k_{0}^{-2}\left(k_{n}^{2}-k_{0}^{2}\right)} u_{n},
$$

where $k_{0}=\omega / c_{0}$ and $c_{0}$ is a reference sound speed. The square root of the operator in Eq. (7) can be approximated by Padé coefficients. ${ }^{37}$ When accurate wavenumbers and mode shapes are available, AMPE then solves for the $u_{n}$ 's for all values of $\theta$ at each range. The necessary wavenumber and mode parameters in Eq. (5) are obtained for the average sound speed profile and the geoacoustic parameters shown by the dark curves in Fig. 4 using COUPLE. The AMPE code was then modified to accept this output from COUPLE and to account for thermocline variations (in addition to bathymetric variations). The code was further modified to compute received complex pressure for construction of the waveguide transfer function for broadband synthesis. a)
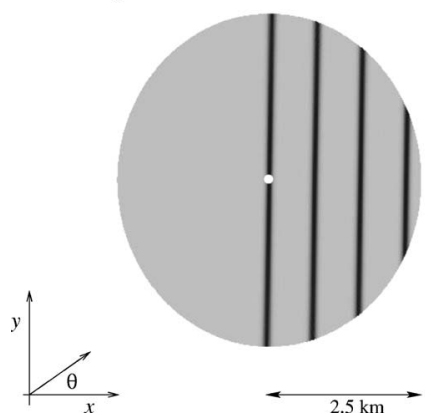

b)

FIG. 8. Top view of portion of 3D simulation environment. Source (white dot) in center of circle. Gray scale shows thermocline variation from nonlinear internal wave model in Eq. (2) using coordinates shown. (a) Internal wave peak near source location produces defocusing of acoustic energy. (b) Source in trough between two internal waves produces acoustic focusing.

\section{THREE-DIMENSIONAL CALCULATION RESULTS}

The adiabatic mode PE method is used to determine the horizontal refraction from nonlinear internal waves past a source at $12 \mathrm{~m}$ depth. Figure 8 shows the circular region used for AMPE calculations. The assumption of linear wave fronts makes it convenient to introduce a Cartesian $(x, y)$ coordinate system originating at the source with the $x$ direction pointing to the right. The nonlinear internal wave model from Eq. (2) is used with $r \rightarrow x$ and $r_{n} \rightarrow x_{n}$ (initial peak locations), $v=0.42 \mathrm{~m} / \mathrm{s}$ is the packet velocity, and $\Lambda_{n}=\Lambda$ is the width of each wave. Shade variations in Fig. 8 represent thermocline depressions from the nonlinear internal waves in Eq. (2). Figure 8(a) shows a situation where an internal wave peak is passing over the source, while Fig. 8(b) shows the source in a trough between two waves.

This coordinate system is also used with the standard $(r, \theta)$ coordinates when performing AMPE calculations. Thus, for our simulations, the nonlinear internal waves propagate in the negative $x$ direction and over the source. To model the situation at the WHOI VLA, we examine acoustic results for a range of angles near $\theta=90^{\circ}$.

\section{A. Single frequency}

Because the upper water depths have higher sound speeds than the lower, acoustic waves refract away from nonlinear internal wave peaks. Thus when the source is positioned as in Fig. 8(a), defocusing of acoustic energy occurs near the $90^{\circ}$ azimuth in our coordinates. When the source is positioned as in Fig. 8(b), acoustic waves refract into the low sound speed trough between nonlinear internal waves and acoustic focusing results. These effects have been predicted using ray theory, ${ }^{3}$ exhibited computationally, ${ }_{14}^{18}$ and mentioned in connection with experimental results. ${ }^{14}$

Figure 9 shows transmission loss results from AMPE computations for three dominant peak frequencies of the airgun source in a $25 \mathrm{~km}$ radius, wedge-shaped region centered at the $90^{\circ}$ azimuth. In all panels, light to dark represents high to low transmission loss, and results for the two situations in Fig. 8 are shown. Figure 9(a) displays calculations for a $32 \mathrm{~Hz}$ source between internal waves and low loss occurs at the peak of the nonlinear internal wave, with somewhat 
(a)

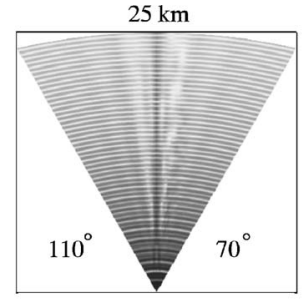

$0 \mathrm{~km}$

(c)

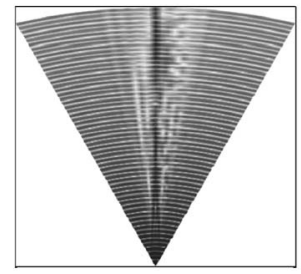

(e)

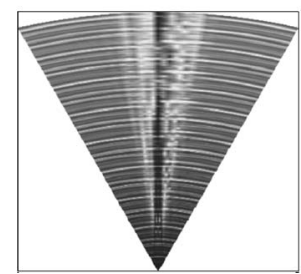

(b)

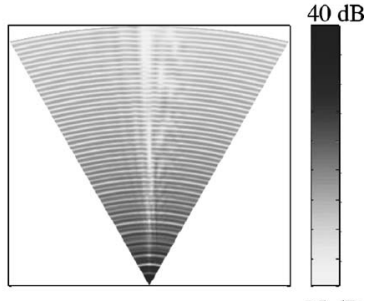

(d)

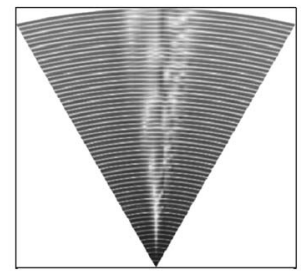

(f)

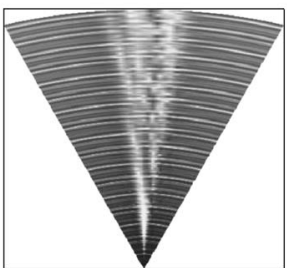

FIG. 9. Wedge-shaped regions are top portions of circles shown in Fig. 8 Contours are $\mathrm{CW}$ results at $19 \mathrm{~m}$ depth from AMPE calculations. Light shades represent high loss, and dark low. Acoustic energy at $32.5 \mathrm{~Hz}$ is (a) focused and (b) defocused. (c), (d) Same for $65 \mathrm{~Hz}$. (e), (f) Same for $95 \mathrm{~Hz}$.

larger losses on either side of the peak. Similar focusing occurs for a $64 \mathrm{~Hz}$ source in Fig. 9(c) and for a $95 \mathrm{~Hz}$ source in Fig. 9(e). At the higher frequencies it is possible to see acoustic rays converging near $15-18 \mathrm{~km}$. Figure 9(b) shows large losses occur along the $90^{\circ}$ azimuth for a $32 \mathrm{~Hz}$ source at a nonlinear internal wave peak. Defocusing is also present in Figs. 9(d) and 9(f) for a 64 and $95 \mathrm{~Hz}$ source. The higher frequencies exhibit ray-like refraction near the $90^{\circ}$ azimuth.

\section{B. Broadband}

AMPE was used to compute the waveguide transfer function near three peak frequencies of 32,64 , and $95 \mathrm{~Hz}$ at all azimuths of the 3D environment. Broadband pulses were obtained using the source representation developed in Ref. 23 and standard Fourier synthesis techniques. Broadband pulses obtained from AMPE from an environment with no internal waves preserve the relevant amplitude and arrival characteristics for the dominant modes of each frequency band shown in Fig. 5.

Simulated broadband pulses from the $87^{\circ}$ azimuth are shown in Fig. 10 for defocused (dark curves) and focused (light curves) situations. Figure 10 shows pulses normalized by the maximum of the defocused signal for (a) full band, and Butterworth filtered signals centered at (b) 32, (c) 64, and (d) $95 \mathrm{~Hz} . I_{T}$ calculations are shown next to each curve. Large $\mathrm{dB}$ differences exist in all frequency bands. Figure 10(b) shows a notable change in the $32 \mathrm{~Hz}$ band second mode arrival. Modal arrival times in Figs. 10(c) and 10(d) show small differences, but the primary effect is the decreased amplitude of focused curves.
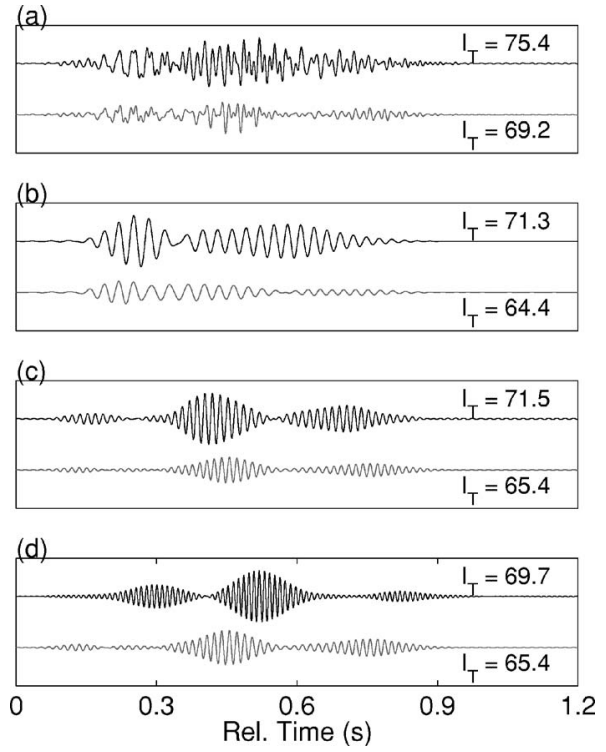

FIG. 10. Broadband AMPE simulations for defocused (dark curve) and focused (light curve) internal wave situations. (a) Full band. (b) Butterworth filter centered at $32 \mathrm{~Hz}$. (c) Same, but filter centered at $64 \mathrm{~Hz}$. (d) Same, but filter centered at $95 \mathrm{~Hz} . I_{T}$ calculations are shown next to each curve and indicate several $\mathrm{dB}$ differences occur between defocused and focused situations.

Figure 11(a) shows Gabor wavelet analysis of the defocused acoustic pulse in Fig. 10(a). The spectrum and scalogram both show expected energy peaks in the 64 and $95 \mathrm{~Hz}$ bands. Figure 11(b) shows the spectrum and scalogram for the focused pulse. The spectrum shows less energy arriving in the $32 \mathrm{~Hz}$ band and more arriving in higher frequency 95 and $120 \mathrm{~Hz}$ bands, consistent with patterns in the data. Timefrequency variations in higher frequency bands are not as prevalent as they appear in data, though modal arrival times are affected. In an adiabatic setting we do not anticipate significant modal interaction. However, Fig. 11 shows that horizontal refraction effects appear to have caused observable time-frequency variations of these pulses and suggests this is a possible mechanism for similar variations in the data.

A comparison between the complete evolution of data and computation acoustic pulses over geotime is shown in Fig. 12. Figure 12(a) shows geotime behavior for 31 consecutive $32 \mathrm{~Hz}$ band pulses recorded at the WHOI VLA phone $2(19 \mathrm{~m})$. Clear amplitude variations occur that correspond to observed fluctuations in $I_{T}$. As geotime increases, both first and second mode amplitudes increase and the second mode tends to arrive closer to the first mode. Once the maximum amplitudes are achieved, the magnitudes of both modes decrease to a minima near minute 12 , then increase, and decrease again, with the second mode arrivals exhibiting a pattern similar to the first several minutes. Figure 12(b) shows 31 simulated geotime minutes at $15 \mathrm{~km}$ range, $19 \mathrm{~m}$ depth, and $87^{\circ}$ azimuth for a packet with $\Lambda=140 \mathrm{~m}, x_{n}$ $=2.3 n \Lambda \mathrm{m}$, and $v=0.42 \mathrm{~m} / \mathrm{s}$. The amplitude variation patterns of both modes are fully consistent with data in Fig. 12(a), as are variations in relative second mode arrival time.

Figure 13 compares pulse-averaged intensity $I_{T}$ for data (dashed curve) at hydrophone $2(19 \mathrm{~m})$ in the $32 \mathrm{~Hz}$ band and the corresponding computation (solid curve with circles) 
(a)
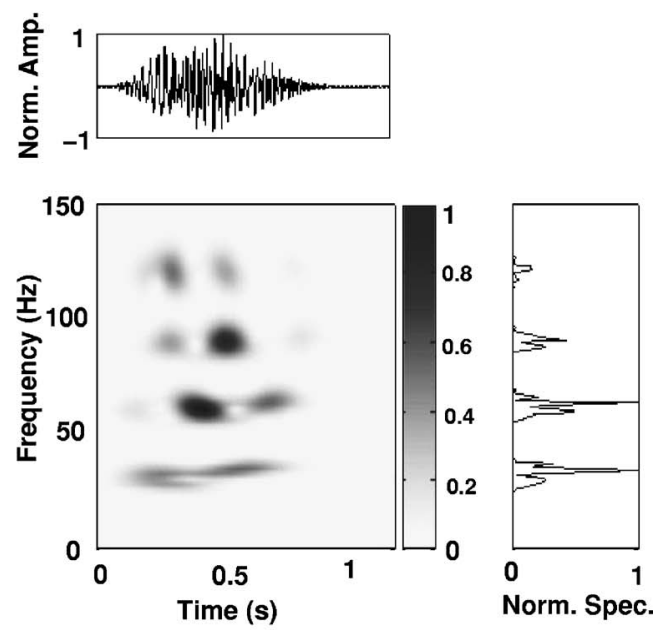

(b)
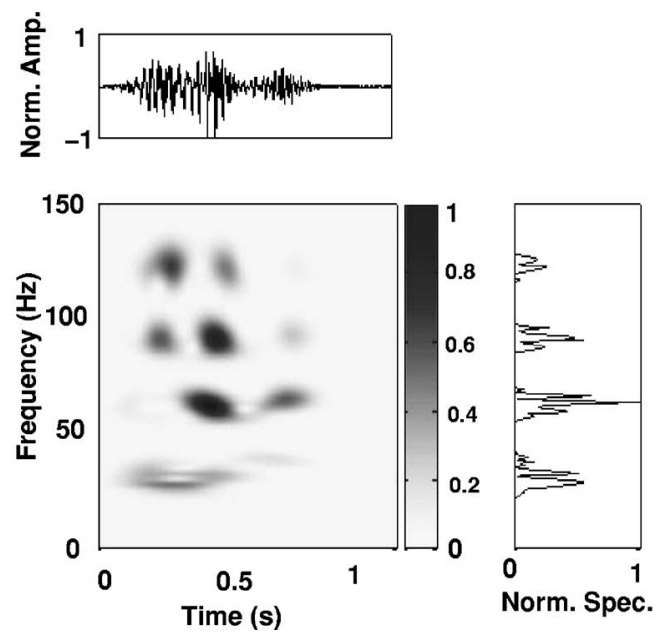

FIG. 11. Gabor wavelet analysis of AMPE simulations at $19 \mathrm{~m}$ depth for (a) focused and (b) defocused pulses in Fig. 10. Focused pulse shows more acoustic energy at $32 \mathrm{~Hz}$, while defocused pulse has more energy in higher frequency bands. These features are consistent with time-frequency analyzed data.

from the $87^{\circ}$ azimuth. The occurrence of maximum and minimum $I_{T}$ values are consistent between data and simulations, as suggested by Fig. 12. The amplitude of the data fluctuations ranges up to nearly $6 \mathrm{~dB}$. The simulation curves have a maximum variation of about $10 \mathrm{~dB}$ and shows three peaks, with the time between peaks essentially the same as the data. This figure clearly shows that the large variations observed in the data can result from horizontal refraction. It is emphasized that the nonlinear internal wave model and parameters are the same as those used for the other southwest track, ${ }^{23}$ although, of course, neither these values nor the model should be considered unique. The main point is that the same ocean environmental characterization can account for the observed acoustic variations on two distinct tracks.

Calculated broadband geotime pulse-averaged intensity variations for the $32 \mathrm{~Hz}$-centered band are visible for up to $\pm 10^{\circ}$ on either side of the $90^{\circ}$ azimuth. In Fig. 14 shadings toward white indicate $I_{T}$ values for a broadband pulse (at any particular geotime and azimuth) that are above the mean, while shadings toward black indicates values below the mean. Two prominent white stripes near $90^{\circ}$ result from fo-
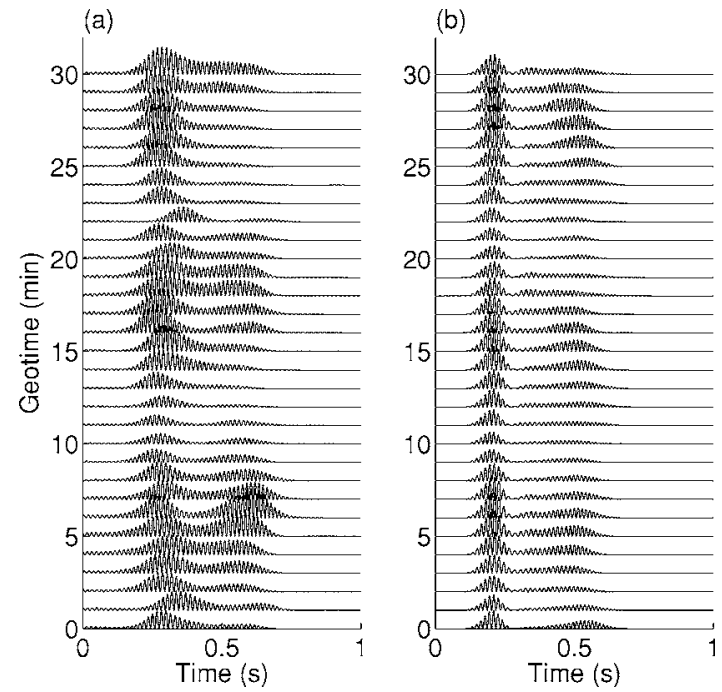

FIG. 12. (a) Pulse time-series data at WHOI phone 2 (19 m depth) in $32 \mathrm{~Hz}$ band for $30 \mathrm{~min}$. Variations in signal amplitude correspond with $I_{T}$ oscillations. Second mode arrival time also varies. (b) Corresponding results from broad-band AMPE simulations centered at $32 \mathrm{~Hz}$ and received at $15 \mathrm{~km}$ on the $87^{\circ}$ azimuth. Amplitude variation of dominant mode arrival times are consistent with data.

cusing and defocusing effects of the nonlinear internal wave channel. The amplitudes of the variations tend to diminish in amplitude further from $90^{\circ}$. The solid curve in Fig. 13 corresponds to the cross section at $87^{\circ}$.

\section{SUMMARY AND DISCUSSION}

Broadband data from the WHOI VLA of SWARM shows time-frequency variation patterns that have similar patterns to those at the NRL VLA. However, pulse-averaged intensity variations at the WHOI VLA have considerably larger amplitudes than those at the NRL array. This suggests that the nonlinear internal wave packet which traverses both tracks has a more substantial influence on the WHOI VLA

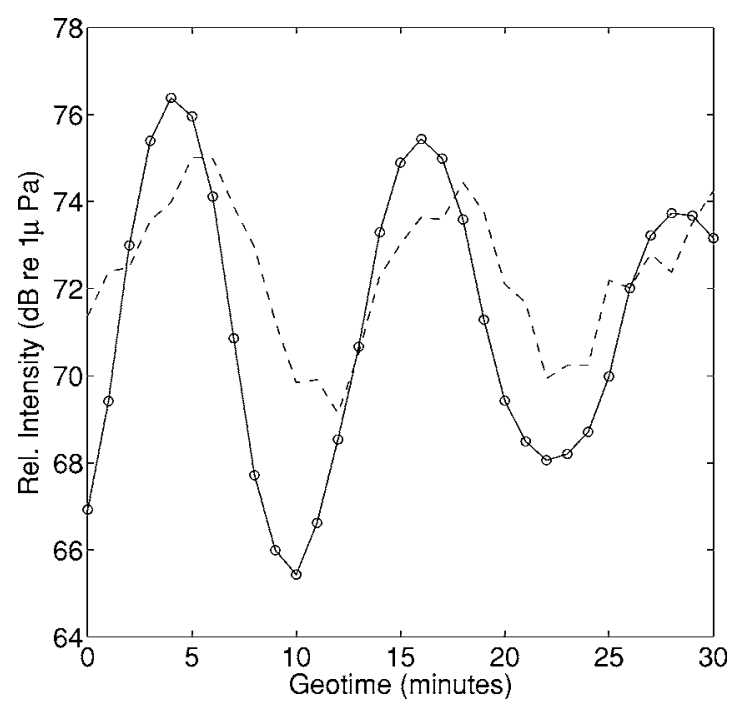

FIG. 13. $I_{T}$ variations for data (dashed curve) and AMPE simulations (solid curve) at phone $2(19 \mathrm{~m}$ depth) for signals centered at $32 \mathrm{~Hz}$. Adiabaticmode horizontal refraction can account for large $I_{T}$ variations observed in data. Peak locations support selected values for internal wave parameters. 


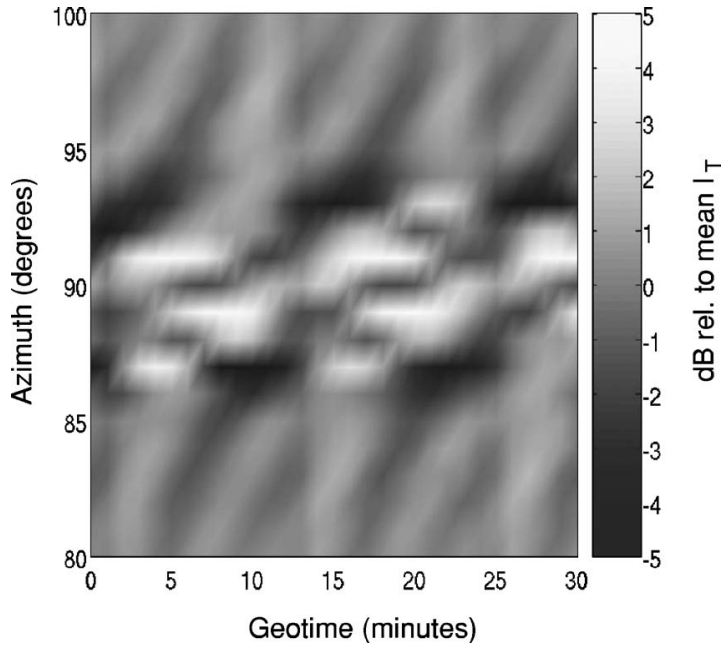

FIG. 14. Contour plot of variations from geotime mean $I_{T}$ vs azimuth and geotime for $32 \mathrm{~Hz}$ broadband computations. Light represents increased $I_{T}$ and dark represents decreased $I_{T}$. Oscillatory patterns arise for at least $10^{\circ}$ on both sides of the $90^{\circ}$ azimuth.

signals than those on the NRL VLA. Traditional 2D PE simulations evidently do not model fluctuations in broadband signals when nonlinear internal wave fronts are nearly parallel to the acoustic propagation direction. In this particular "high incidence angle" situation, 2D mechanisms such as mode coupling and internal wave-acoustic resonance (which are effective for lower incidence angles) apparently cannot produce sufficiently strong intensity variations. For high incident angles the environmental gradients are large enough that significant energy propagates out of vertical planes, as previous numerical computations have demonstrated. Consequently, acoustic horizontal refraction must be taken into account for the low frequency bands in this experiment.

Rough theoretical estimates calculated for the critical deflection angle and focal range of horizontally refracted acoustic rays are consistent with both experimental observations and model calculations. Similarly, an acoustic intensity amplitude estimate also provides overall agreement. The effect of small curvature of nonlinear internal wave fronts is addressed briefly.

Calculations using the adiabatic mode PE for the WHOI track show focusing and defocusing of acoustic energy as nonlinear internal waves pass a source. Large azimuthal variations occur in $\mathrm{CW}$ transmission loss for 32, 65, and $95 \mathrm{~Hz}$. Broadband synthesis for dominant frequency bands in the airgun source show that variations of about $6 \mathrm{~dB}$ in pulse-averaged $I_{T}$ arise using a model packet of nonlinear internal waves. Fluctuations in the time-frequency behavior of these calculations are also consistent with data from the WHOI VLA. The principal result is that the propagation model calculations provide strong evidence that observations at the WHOI VLA of SWARM show the influence of horizontal refraction due to nonlinear internal waves. To our knowledge, this is the first evidence of this type for threedimensional refraction effects in the literature. Results in Ref. 41, which emphasize horizontal rays and vertical modes rather than a computational model for the track, conclude that horizontal refraction is the likely cause of observed in- tensity variations. A critical feature of the analysis here is that the "tuning" is done for the ocean environmental model of the nonlinear internal waves. Exactly the same assumptions and parameter values are used as for the previous analysis of the 4 August 1995 packet along a distinct acoustic track. ${ }^{23}$ This does not imply that the environmental model is either optimal or unique, but rather that the mechanisms behind the acoustic variability on both tracks appear to be robust. Indeed, frequency-dependent results and related analysis reiterate the robustness of horizontal refraction effects on the WHOI track.

Three-dimensional broadband calculations are computationally intensive even with the relatively efficient AMPE algorithm, so geotime simulations remain to be performed for higher frequency bands from the airgun. A more complete sensitivity study of geoacoustic and internal wave parameters on horizontal refraction effects would be worthwhile. While the assumption of linear internal wave fronts is reasonable, ${ }^{24}$ further investigation of how wave front curvature affects horizontal refraction and subsequent intensity and timefrequency variations would extend this work. The interaction between 2D mechanisms such as mode-coupling or resonance and horizontal refraction, which is an inherently $3 \mathrm{D}$ effect, is an important question. Earlier work ${ }^{8}$ suggests that this type of interaction may occur for incidence angles above about $70^{\circ}$. Due to the adiabatic nature of the $3 \mathrm{D}$ model in this study, this question was not addressed. However, it would be interesting to obtain estimates of the azimuths where mode coupling effects become negligible and horizontal refraction effects become significant. Finally, seafloor bathymetry interactions with nonlinear internal wave packets are not addressed here, and recent work suggests that this combination can be important. ${ }^{42}$

\section{ACKNOWLEDGMENTS}

The authors gratefully acknowledge Dr. Yongke $\mathrm{Mu}$ for assistance reading the acoustic data and Dr. Michael Collins for providing the original AMPE code. We also acknowledge the work of the scientists associated with the SWARM 95 experiment which received support from NRL base funds. This work was supported by an ONR Ocean Acoustics Graduate Traineeship Award and by ONR grants to Rensselaer, the University of Delaware, and the Woods Hole Oceanographic Institution. The principal contributions of this work were taken from a Ph.D. thesis by S. D. Frank, submitted to Rensselaer Polytechnic Institute in July 2003. This is WHOI contribution No. 11257.

\footnotetext{
${ }^{1}$ J. Zhou, X. Zhang, and P. H. Rogers, "Resonant interaction of sound waves with internal solitons in the coastal zone," J. Acoust. Soc. Am. 90, 2042-2054 (1991).

${ }^{2}$ A. Y. Shmelerv, A. A. Migulin, and V. G. Petnikov, "Horizontal refraction of low-frequency acoustic waves in the Barents Sea stationary acoustic track experiment," J. Acoust. Soc. Am. 92, 1003-1007 (1992).

${ }^{3}$ B. G. Katsnel'son and S. A. Pereselkov, "Low-frequency horizontal acoustic refraction caused by internal wave solitons in a shallow sea," Acoust. Phys. 46, 779-788 (2000).

${ }^{4}$ J. F. Lynch, M. H. Orr, and S. N. Wolf, "Low frequency acoustic propagation through shallow water internal waves," in Sound Propagation Through Internal Waves (unpublished).

${ }^{5}$ J. R. Apel, M. Badiey, C.-S. Chiu, S. Finette, R. H. Headrick, J. Kemp, J.
} 
F. Lynch, A. E. Newhall, M. H. Orr, B. H. Pasewark, D. Tielbuerger, A Turgut, K. von der Heydt, and S. N. Wolf, "An overview of the 1995 SWARM shallow-water internal wave acoustic scattering experiment," IEEE J. Ocean. Eng. 22, 465-499 (1996).

${ }^{6}$ R. H. Headrick, J. F. Lynch, J. N. Kemp, A. E. Newhall, K. von der Heydt, J. R. Apel, M. Badiey, C.-S. Chiu, S. Finette, M. H. Orr, B. Pasewark, A. Turgut, S. N. Wolf, and D. Tielbuerger, "Acoustic normal mode fluctuation statistics in the 1995 SWARM internal wave scattering experiment," J. Acoust. Soc. Am. 107, 201-220 (2000).

${ }^{7}$ D. Rouseff, A. Turgut, S. N. Wolf, S. Finette, M. H. Orr, B. H. Pasewark, J. R. Apel, M. Badiey, C.-S. Chiu, R. H. Headrick, J. F. Lynch, J. N. Kemp, A. E. Newhall, K. von der Heydt, and D. Tielbuerger, "Coherence of acoustic modes propagating through shallow water internal waves," J. Acoust. Soc. Am. 111, 1655-1666 (2002).

${ }^{8}$ J. C. Preisig and T. F. Duda, "Coupled acoustic mode propagation through continental-shelf internal solitary waves," IEEE J. Ocean. Eng. 22, 256269 (1997).

${ }^{9}$ T. F. Duda and J. C. Preisig, "A modeling study of acoustic propagation through moving shallow water solitary wave packets," IEEE J. Ocean Eng. 24, 16-32 (1999)

${ }^{10}$ D. Tielbuerger, S. Finette, and S. N. Wolf, "Acoustic propagation through an internal wave field in a shallow water waveguide," J. Acoust. Soc. Am. 101, 789-808 (1997).

${ }^{11}$ M. Badiey, Y. Mu, J. F. Lynch, J. R. Apel, and S. N. Wolf, "Temporal and azimuthal dependence of sound propagation in shallow water with internal waves,” IEEE J. Ocean. Eng. 27, 117-129 (2002).

${ }^{12}$ D. Rubenstein and M. H. Brill, "Acoustic variability due to internal waves and surface waves in shallow water," in Ocean Variability and Acoustic Propagation, edited by J. Potter and A. Warn-Varnas (Kluwer Academic, Boston, 1991), pp. 215-228.

${ }^{13}$ D. Rubenstein, "Observations of cnoidal internal waves and their effect on acoustic propagation in shallow water," IEEE J. Ocean. Eng. 24, 346-357 (1999).

${ }^{14}$ O. C. Rodriguez, S. Jesus, Y. Steephan, X. Demoulin, M. Porter, and E. Coelho, "Nonlinear soliton interaction with acoustic signals: Focusing effects," J. Comput. Acoust. 8, 347-363 (2000).

${ }^{15}$ J. F. Lynch and P. H. Dahl, "Overview of ASIAEX field experiments in the South and East China Seas," J. Acoust. Soc. Am. 112, 2360 (2002).

${ }^{16} \mathrm{R}$. Burridge and H. Weinberg, "Horizontal rays and vertical modes," in Wave Propagation and Underwater Acoustics, Lecture Notes in Physics, Vol. 70, edited by J. Keller and J. S. Papadakis (Springer, New York, 1977), Chap. 2.

${ }^{17}$ Y. A. Kravtsov, V. M. Kuzkin, and V. G. Petnikov, "Perturbation calculation of the horizontal refraction of sound waves in a shallow sea," Sov. Phys. Acoust. 30, 45-47 (1984).

${ }^{18}$ R. Oba and S. Finette, "Acoustic propagation through anisotropic internal wave fields: Transmission loss, cross-range coherence, and horizontal refraction," J. Acoust. Soc. Am. 111, 769-784 (2002).

${ }^{19} \mathrm{~S}$. Finette and R. Oba, "Horizontal array beamforming in an azimuthally anisotropic internal wave field," J. Acoust. Soc. Am. 114, 131-144 (2003)

${ }^{20}$ K. B. Smith, C. W. Miller, A. F. D’Agostino, B. Sperry, J. H. Miller, and G. R. Potty, "Three-dimensional propagation effects near the Mid-Atlantic Bight shelf break (L)," J. Acoust. Soc. Am. 112, 373-376 (2002).

${ }^{21}$ R. F. Henrick, M. J. Jacobson, and W. L. Siegmann, "General effects of currents and sound-speed variations on short-range acoustic transmission in cyclonic eddies," J. Acoust. Soc. Am. 67, 121-134 (1980).

${ }^{22}$ K. G. Hamilton, W. L. Siegmann, and M. J. Jacobson, "Simplified calculation of ray-phase perturbations due to ocean-environment variations," J.
Acoust. Soc. Am. 67, 1193-1206 (1980)

${ }^{23}$ S. D. Frank, M. Badiey, J. F. Lynch, and W. L. Siegmann, "Analysis and modeling of broadband airgun data influenced by nonlinear internal waves," J. Acoust. Soc. Am. 116, 3404-3422 (2004).

${ }^{24}$ A. K. Liu, "Analysis of nonlinear waves in the New York Bight," J. Geophys. Res. 93, 12317-12329 (1988).

${ }^{25}$ National Geophysical Data Center, NOAA, Hydrographic Survey Data, Vol. 1, version 3.3.

${ }^{26}$ J. D. Milliman, A. Jiezao, L. Anchun, and J. I. Ewing, "Late quaternary sedimentation on the outer and middle New Jersey Continental shelf: Result of two local deglaciations?," J. Geol. 98, 966-976 (1990).

${ }^{27} \mathrm{~J}$. M. Hovem and A. Kristensen, "Reflection loss at a bottom with a fluid sediment layer over a hard solid half-space," J. Acoust. Soc. Am. 92, 335-340 (1992).

${ }^{28} \mathrm{C}$. T. Tindle and Z. Y. Zhang, "An equivalent fluid approximation for a low shear speed ocean bottom," J. Acoust. Soc. Am. 91, 3248-3256 (1992).

${ }^{29}$ A. Turgut and S. N. Wolf, "Matched-field inversion of seabed geoacoustic properties complemented by chirp sonar surveys," J. Acoust. Soc. Am. 110, 2661(A) (2001).

${ }^{30}$ M. D. Collins, "RAMGEO 1.5," URL ftp://albacore.nrl.navy.mil/RAM.

${ }^{31}$ B. G. Katsnel'son and S. A. Pereselkov, "Resonance effects in sound scattering by internal wave packets in a shallow sea," Acoust. Phys. 44, 684-689 (1998)

${ }^{32}$ R. B. Evans, "A coupled mode solution for acoustic propagation in a waveguide with stepwise depth variations of a penetrable bottom," J. Acoust. Soc. Am. 74, 188-195 (1983), URL http://oalib.saic.com/Modes/ couple/.

${ }^{33}$ I. Rozenfeld, W. M. Carey, P. G. Cable, and W. L. Siegmann, "Modeling and analysis of sound transmission in the Strait of Korea," IEEE J. Ocean. Eng. 26, 809-819 (2001).

${ }^{34} \mathrm{Z}$. Y. Zhang and C. T. Tindle, "Improved equivalent fluid approximations for a low shear speed ocean bottom," J. Acoust. Soc. Am. 98, 3391-3396 (1995).

${ }^{35}$ F. B. Jensen, W. A. Kuperman, M. B. Porter, and H. Schmidt, Computational Ocean Acoustics (Springer, Berlin, 2000).

${ }^{36}$ J. F. Lynch, J. A. Colosi, G. Gawarkiewicz, T. F. Duda, A. D. Pierce, M. Badiey, B. Katsnelson, J. E. Miller, W. L. Siegmann, C. Chiu, and A. Newhall, "Inclusion of finescale coastal oceanography and 3-D acoustics effects into the ESME sound exposure model," IEEE J. Ocean. Eng. (submitted).

${ }^{37}$ M. D. Collins, "The adiabatic mode parabolic equation," J. Acoust. Soc. Am. 94, 2269-2278 (1993)

${ }^{38} \mathrm{~B}$. Coury, "Energy conservation and interface conditions for parabolic approximations to the Helmholtz Equation," Ph.D. thesis, Rensselaer Polytechnic Institute, 1996.

${ }^{39}$ B. G. Katsnel'son, S. A. Pereselkov, V. G. Petnikov, K. D. Sabinin, and A. N. Serebryanyi, "Acoustic effects caused by high-intensity internal waves in a shelf zone," Acoust. Phys. 47, 424-429 (2001).

${ }^{40} \mathrm{~K}$. B. Smith, "A three-dimensional propagation algorithm using finite azimuthal aperture," J. Acoust. Soc. Am. 106, 3231-3239 (1999).

${ }^{41}$ M. Badiey, B. G. Katsnelson, J. F. Lynch, S. Pereselkov, and W. L. Siegmann, "Measurement and modeling of three-dimensional sound intensity variations due to shallow-water internal waves," J. Acoust. Soc. Am. 90, 613-625 (2005).

${ }^{42}$ A. D. Pierce and J. F. Lynch, "Whispering-gallery-mode trapping of sound in shallow water between an up-slope region and internal wave solitons," J. Acoust. Soc. Am. 113, 2279(A) (2003). 